\title{
Development of Monitoring Software for Optical Sensor Networks
}

\author{
M. F. M. Silva e A. D. F. Santos
}

\begin{abstract}
This work presents a monitoring software responsible for processing, persistence and visualization of the data obtained from monitoring realized through optical sensor networks. This software is consist of a data acquisition application (InterAB) and a Web application. To demonstrate its operation, a experiment is presented and discussed, showing results indicating the robustness of the monitoring software in terms of fulfill the proposed requirements for the software.
\end{abstract}

Keywords-InterAB; Web application; Optical sensor networks.

\section{INTRODUCTION}

The monitoring realized through optical sensor networks has shown great expansion in civil engineering applications, oil and gas, composites, smart structures, power transmission networks and many others.

The quantities of interest monitored through optical sensor networks can be temperature, strain, acceleration, among others [1]. Certainly each one of these quantities depend on appropriate optical sensors based, for example, in Fiber Bragg Gratings (FBG), Long Period Gratings (LPG) or Fabri Perot filters [2]. Due to complexity and diversity of these systems, becomes important the flexible and extensible computer software development that allows the processing, persistence and visualization of data obtained from monitoring realized through optical sensor networks.

This work focuses on the development of an monitoring software, integrated by acquisition system (InterAB) and visualization system (Web application). Nevertheless, the FS2200 optical interrogator, FS6300 temperature and FS6200 strain FBG sensors were used as a case study, both manufactured by FiberSensing.

The paper is organized as follows: the Section II introduces the sensing principles using FBG optical sensors; the Section III focuses on the operation of the InterAB system and Web application; the Section IV presents and discussed the results; the conclusion is presented in Section V.

\section{STRAIN AND TEMPERATURE SENSING PRINCIPLES WITH FBG OPTICAL SENSORS}

An FBG is a type of Bragg reflector constructed inside an optical fiber, able to reflect a range of wavelengths, keeping relatively transparent to the rest of the spectrum [3]. The sensing function of an FBG derives from the sensitivity

Moisés Felipe M. da Silva e Adam Dreyton F. dos Santos, Applied Electromagnetics Laboratory, Federal University of Pará, Belém, Pará, Brazil. E-mails: moises.felipe@icen.ufpa.bre adam.santos@itec.ufpa.br. This work was partially funded by $\mathrm{CNPq}$, CAPES and Brazilian Power Transmission Companies (TBE). of the refractive index and grating period to externally applied mechanical or thermal perturbations [4]. The strain field affects the response of an FBG directly, through the expansion and compression of grating pitch size and through the strain-optic effect, i.e., the strain-induced modification of the refractive index. The temperature sensitivity of an FBG occurs principally through the effect on the induced refractive index change and, to a lesser extent, on the thermal expansion coefficient of the fiber [5].

Thus, the peak reflected wavelength shifts by an amount $\Delta \lambda_{B}$ in response to strain $\varepsilon$ and temperature change $\Delta T$ as given by [5]

$$
\frac{\Delta \lambda_{B}}{\lambda_{B}}=P_{e} \varepsilon+\left[P_{e}\left(\alpha_{s}-\alpha_{f}\right)+\varsigma\right] \Delta T,
$$

where $P_{e}$ is the strain-optic coefficient; $\alpha_{s}$ and $\alpha_{f}$ are the thermal expansion coefficients of any fiber bonding material and of the fiber itself, respectively; and $\varsigma$ is the thermo-optic coefficient.

\section{INTERAB SYSTEM AND WEB APPLICATION}

The InterAB system performs data acquistion and subsequent data filtering. The software establishing communication via TCP/IP socket with FS2200 optical interrogator. The Inter $\mathrm{AB}$ sends a command string to the interrogator, returning the peak samples of the FBG sensors. These peaks are persisted by InterAB in a MySQL relational database. The Web Application, which uses the Apache Tomcat server, load the data from the database and present the variations of peaks through of the time in form of graphs generated by PrimeFaces framework. The simplified operation of both systems is shown in Figure 1.

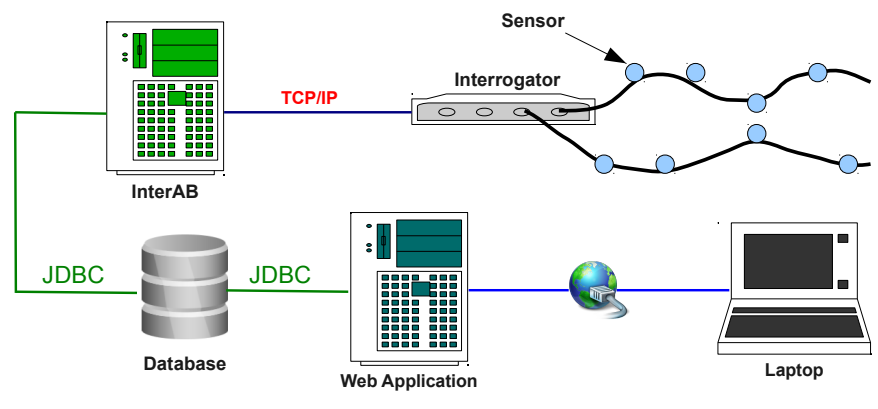

Fig. 1. InterAB system performing data acquisition from optical sensor networks and Web application load these data to present them. 
The Web system has a part of descrip of project open for tzhe public, and another part visible only after login be effected, giving access to the graphs and network topology.

\section{RESUlts}

Aiming to present the main features of the InterAB system integrated with Web application, a experiment using sensors based in Fiber Bragg Gratings and a optical interrogator were realized. The set up for the experiment is presented in Figure 2. This network topology presents three FS6300 FBG temperature sensor in the first three channels of the FS2200 optical interrogator and a FS6200 FBG strain sensor in the last channel.

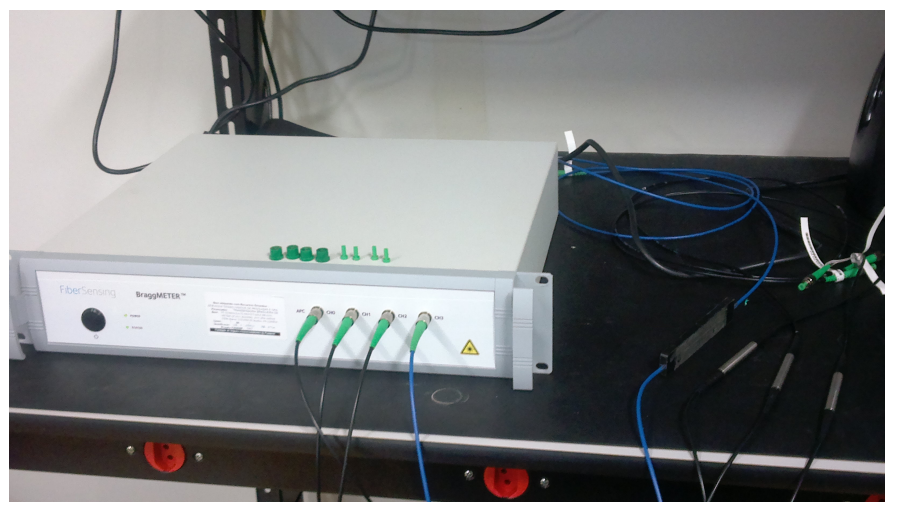

Fig. 2. Scheme set up for experiment with a optical sensor network.

In Figure 3 we can see the network topology recognized through InterAB system and rendered by Web application, displaying the interrogator and the sensors connected in their channels.

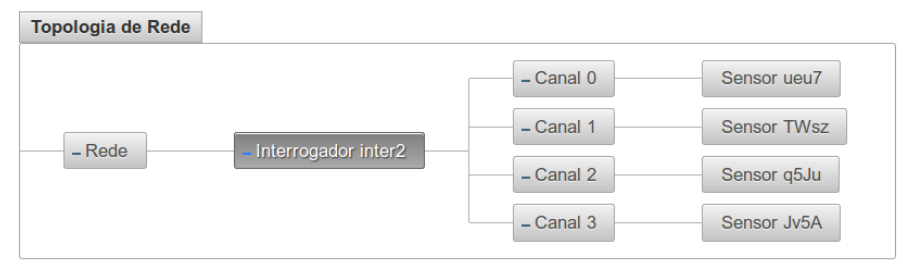

Fig. 3. Optical sensor network topology.

The experiment aims to show how the InterAB system performs filtering of readings generated by optical sensors and their exhibition by Web application. The variation in peak wavelengths of the sensors $0.001 \mathrm{~nm}$ was chosen due to the fact allow view in short-term temperature changes with a reasonable number of frames collected from the FS2200 optical interrogator. The graphics related to temperature and strain sensors show us the variation of the data collected by Inter $\mathrm{AB}$ system, being updated automatically with the new last six samples saved in database. Each curve refers to a different sensor in the topology. The horizontal axis corresponds to the time of data collection, while the vertical axis represents shift wavelength converted in their quantity corresponding using a simple equation which can be linear or quadratic depending on the sensor type. The data collected by InterAB could be visualized through Web application in Figures 4 and 5.

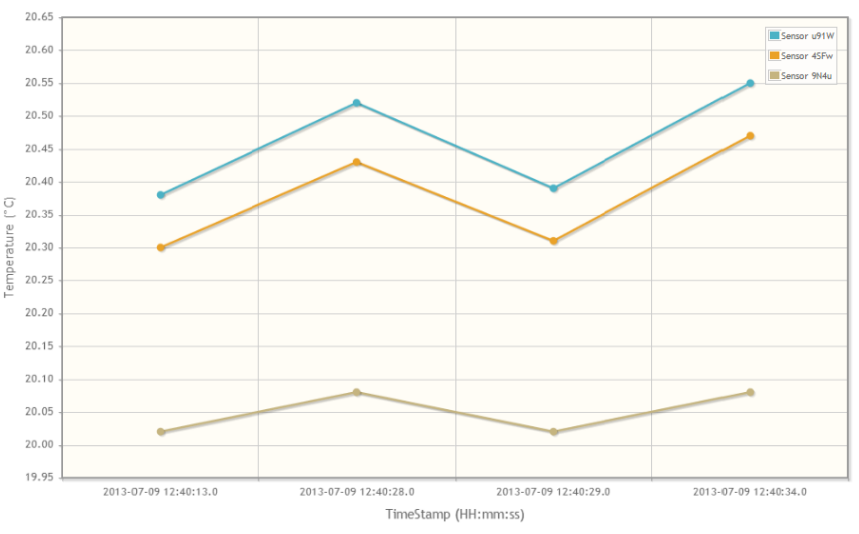

Fig. 4. Visualization of temperature monitoring in Web application.

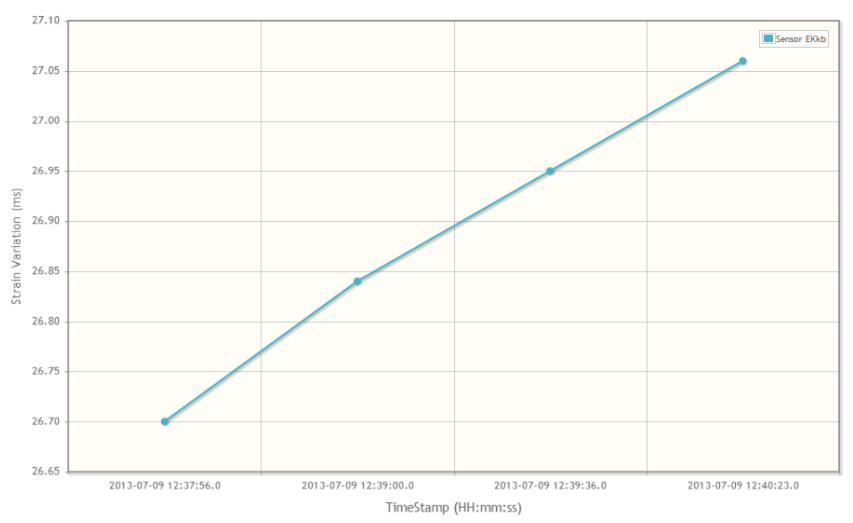

Fig. 5. Visualization of strain monitoring in Web application.

\section{CONCLUSION}

This paper was presented the monitoring software for optical sensor networks in order to process, persist and visualization these data. The experiment demonstrated the robustness and flexibility of both applications (InterAB and Web application) that integrated this software. These applications could be easily adapted for others models of interrogators and sensors.

\section{REFERENCES}

[1] H. R. Alemohammad, E. Foroozmehr, B. S. Cotten, and E. Toyserkani. A dual-parameter optical fiber sensor for concurrent strain and temperature measurement: Design, fabrication, packaging, and calibration. Journal of Lightwave Technology, 31(8):1198-1204, 2013.

[2] Junjie Bai, Damei Zhao, and Xiuyan Zhang. Structural health monitoring of smart civil structures based on fiber bragg grating sensing technology. In 2nd International Conference on Artificial Intelligence, Management Science and Electronic Commerce (AIMSEC), pages 635-638, 2011.

[3] P. F. C. Antunes. "Sensores ópticos para monitorização dinâmica de estruturas". PhD thesis, Universidade de Aveiro, Jul 2011.

[4] Peng Wang, Jie Liu, Fangchao Song, and Hong Zhao. Quasi-distributed temperature measurement for stator bars in large generator via use of fiber bragg gratings. In 6th International Forum on Strategic Technology (IFOST), volume 2, pages 810-813, 2011.

[5] S. Yin, P. B. Ruffin, and F. T. S. Yu. Fiber optic sensors. CRC Press, second edition, 2008. 